\title{
Multi-modal measurement of the myelin-to-axon diameter g-ratio in preterm-born neonates and adult controls
}

\author{
Andrew Melbourne ${ }^{1}$, Zach Eaton-Rosen ${ }^{1}$, Enrico De Vita ${ }^{3}$, Alan Bainbridge ${ }^{4}$, \\ M. Jorge Cardoso ${ }^{1}$, David Price ${ }^{4}$, Ernest Cady ${ }^{4}$, Giles S. Kendall ${ }^{2}$, Nicola J. \\ Robertson $^{2}$, Neil Marlow ${ }^{2}$, and Sebastien Ourselin ${ }^{1}$ \\ ${ }^{1}$ Centre for Medical Image Computing, University College London, UK \\ ${ }^{2}$ Academic Neonatology, EGA UCL Institute for Women's Health, London, UK \\ ${ }^{3}$ Academic Neuroradiological Unit, UCL Institute of Neurology, London, UK \\ ${ }^{4}$ Medical Physics, University College Hospital, London, UK
}

\begin{abstract}
Infants born prematurely are at increased risk of adverse functional outcome. The measurement of white matter tissue composition and structure can help predict functional performance and this motivates the search for new multi-modal imaging biomarkers. In this work we develop a novel combined biomarker from diffusion MRI and multi-component $\mathrm{T} 2$ relaxation measurements in a group of infants born very preterm and scanned between 30 and 40 weeks equivalent gestational age. We also investigate this biomarker on a group of seven adult controls, using a multi-modal joint model-fitting strategy. The proposed emergent biomarker is tentatively related to axonal energetic efficiency (in terms of axonal membrane charge storage) and conduction velocity and is thus linked to the tissue electrical properties, giving it a good theoretical justification as a predictive measurement of functional outcome.
\end{abstract}

\section{Introduction}

Very preterm birth (birth at less than 32 weeks completed gestational age) occurs at a time of rapid neurological development. During the period between 30 and 40 weeks gestation, the cerebral cortex of the brain changes appearance from a smooth, unfolded state to one in which many tertiary folds are present in advance of birth. In the developing white matter, processes leading to myelination progress in an established spatial pattern, ascending into the corticospinal tracts from as early as 30 weeks gestation and progressing from this region anterior and posterior over the first few months of life [2]. Infants born very preterm are at increased risk of adverse neurodevelopmental outcome [1] and this is believed to be related to delay or disruption to normal developmental processes during this crucial time. The reproducibility of the myelin developmental trajectory means that if myelin content can be measured, myelin location and quantity might be predictive of delays in the subsequent myelination process and thus of neurological developmental delay in infancy. A number of Magnetic Resonance 
(MR) based techniques have been used to infer myelin content including magnetisation transfer [3] and T2 relaxometry [4]. Diffusion Weighted MRI (DWI) is sensitive to local structure, but the short T2 of the myelin signal component means that DWI is generally non-specific to myelin, although its presence will have an occult influence on parameters derived from a diffusion model. In this work we estimate the myelin water signal from multi-echo multi-component T2 relaxometry [4] and combine this with the intra-axonal volume signal measured by a multi-compartment DWI measurement [5]; this imaging combination can be used to define a novel in vivo imaging biomarker that makes use of the structural sensitivity, but myelin inspecificity, of DWI with the high myelin specificity, but structural insensitivity of multi-echo T2 relaxometry. Previous work has combined similar measurements in a non-quantitative framework [3] but here we formalise this work and make use of different image acquisitions to obtain a quantitative imaging biomarker, namely the internal axon diameter to myelinated axon diameter g-ratio [6]. Since this measurement has the potential to map the electrical properties of axons, it may be correlated with specific functional measurements. Magnetic Resonance Spectroscopy (MRS) studies have demonstrated changes in white matter metabolism with brain development including an increase in N-Acetylaspartate (NAA) with progressing brain maturity, and a decrease in choline (Cho). The amino acid NAA is synthesised primarily in neuronal (including axonal) mitochondria and is therefore likely to have a relationship to cell energy turnover [8]. In normal brain development, Cho related species incorporate into macromolecules during myelination and become invisible to MRS, thus enhancing the observed NAA/Cho ratio.

Here we investigate how the myelin water fraction and the measured g-ratio vary in preterm-born neonates between 30-40 weeks equivalent gestational age (EGA) and compare this to measurement of the g-ratio in an adult control population. Work of this type, linking functional performance with structural measurement; is particularly salient for preterm-born neonatal cohorts for which accurate prediction of function is crucial to providing effective intervention.

\section{Methods}

Data Imaging data were acquired for 11 preterm infants (one with longitudinal data at 29.4 and 40.7 weeks EGA) (mean birth gestation $=25.1 \pm 0.7 \mathrm{wks}$ ) on a 3 T Phillips Achieva. Diffusion weighted imaging was acquired over 16 directions at a b-value of $750 \mathrm{~s} . \mathrm{mm}^{-2}$ and 32 directions at $b=2000 \mathrm{~s} . \mathrm{mm}^{-2}$ at resolution $2 \mathrm{~mm}$ isotropic. 32-echo multi-component quantitative T2 imaging was acquired at $1.2 \times 1.2 \times 3 \mathrm{~mm}^{3}$ resolution using a GraSE acquisition at $12 \mathrm{~ms}$ TE. In addition, proton Magnetic Resonance Spectroscopy (MRS) was acquired using water suppressed Point Resolved Spectroscopy (PRESS; TR/TE 2288/288ms) with a $14 \times 13 \times 11 \mathrm{~mm}^{3}$ voxel in the left posterior white matter. Spectra were analysed using the AMARES algorithm in the jMRUI spectroscopy package. Peak-area ratios of Cho/total creatine $(\mathrm{Cr}), \mathrm{NAA} / \mathrm{Cho}$, and $\mathrm{NAA} / \mathrm{Cr}$, were calculated. Corticospinal tract (CST) masks between brainstem and the posterior limb of 
the internal capsule (PLIC) are manually delineated on Fractional Anisotropy maps defined by DWI.

Data were also acquired for seven adult control subjects (Mean age $=32.8$ years, range $=23-54$ years) on a $3 \mathrm{~T}$ Siemens Trio. In addition to T1 weighted MPRAGE data at $0.9 \mathrm{~mm}$ isotropic, diffusion weighted imaging was acquired over 8 directions at a b-value of $300 s . \mathrm{mm}^{-2}(1 \mathrm{x} \mathrm{b0}), 32$ directions at a b-value of $700 \mathrm{~s} . \mathrm{mm}^{-2}(4 \mathrm{x} \mathrm{b0})$ and 64 directions at $b=2000 \mathrm{~s} . \mathrm{mm}^{-2}(8 \mathrm{x} \mathrm{b0})$ at resolution $2.5 \mathrm{~mm}$ isotropic. Multi-component quantitative T2 imaging was acquired at 21 echo times at $2.5 \mathrm{~mm}$ isotropic resolution using an EPI read-out. Echo times were finely sampled between $(19-52 \mathrm{~ms}, \mathrm{n}=15)$ and coarsely sampled after $(57-150 \mathrm{~ms}$, $\mathrm{n}=6$ ) to maximise sensitivity to the rapid decay times of myelin-associated water.

Multi-component T2 Relaxometry We assume that the tissue composition can be described by a discrete number of compartments each with it's own associated T2 distribution undergoing exponential decay. Of importance in this model is the detection of signal with $\mathrm{T} 2<50 \mathrm{~ms}$ which is associated with a water signal that is closely interacting myelin. This provides an indirect measurement of myelin content, termed the myelin water fraction, $v_{m w f}$ defined as the sum of all component magnitudes, $m(\mathrm{~T} 2)$, with a T2 $<50 m s$ [4]. In the case of the neonatal data, which uses a multiply refocused echo train and is susceptible to B1-inhomogeneity, T2 component fitting was carried out using the extended phase graph algorithm (EPG) [4] to extract a short component from 20 logspaced components (15-2000ms), used to infer the $v_{m w f}$ given an estimate of the local applied flip angle $\alpha(1)$.

$$
S_{b}(n)=\sum_{n=1}^{20} m\left(\mathrm{~T} 2_{n}\right) \operatorname{EPG}\left(n, \mathrm{TE}, \mathrm{T} 2_{n}, \alpha\right)
$$

In the case of the adult data, using a series of separate echos, T2 component fitting was carried out using a non-linear least squares fit to extract three components of $\mathrm{T} 2=\{20,80,110\} \mathrm{ms}$ from which the short $\mathrm{T} 2$ is used to infer a $v_{m w f}$ and the long component a free-fluid space $v_{i s o}$. The relatively short third T2 is chosen empirically to absorb non-solid tissue. The method makes use of a three-class tissue segmentation (grey/white matter and cerebrospinal fluid) to initialise the algorithm with a white matter probability of myelin of $25 \%$ (2).

$$
S_{b}(\mathrm{TE})=S_{b 0}\left[v_{m w f} e^{-\mathrm{TE} / 20 \mathrm{~ms}}+v_{\text {tissue }} e^{-\mathrm{TE} / 80 \mathrm{~ms}}+v_{i s o} e^{-\mathrm{TE} / 110 \mathrm{~ms}}\right]
$$

Multi-compartment Diffusion Weighted Imaging We fit a multi- compartment signal model to the multi-shell data using non-linear least squares specifically to estimate an intra-axonal volume fraction [7].

$$
S_{a}(b, \mathbf{x})=S_{a 0}\left[v_{i s o}^{\prime} e^{-b d_{i s o}}+v_{i n}^{\prime} \int f(\mathbf{n}, \gamma) e^{-b d_{\| \mid}(\mathbf{x} \cdot \mathbf{n})} d \Omega+v_{e x}^{\prime} e^{-b \mathbf{x} D^{*} \mathbf{x}}\right]
$$


Briefly. the signal model attributes the white matter signal measured by DWI to three compartments; an intra-axonal space $v_{i n}^{\prime}$, and extra-axonal space $v_{e x}^{\prime}$ and a free-isotropic space $v_{i s o}^{\prime}$ [5]. Given the experimental b-value, $b$, and gradient direction, $\mathbf{x}$, the signal from the intra-axonal and extra-axonal spaces is coupled by a specific distribution, $f(\mathbf{n}, \gamma)$, which is assumed to represent axonal dispersion; formally a Watson distribution of oblateness $\gamma$, varying between 0 , for highly oriented axons, up to 1 when there is no preferred structural orientation [7]. A principal diffusion direction incorporated into the extra-axonal diffusion tensor $D^{*}$ can be defined by two angular parameters $\{\theta, \phi\}$. Lastly, $d_{\|}$and $d_{\text {iso }}$ describe parallel (to the principal direction) and isotropic diffusivities respectively.

Estimating the myelin-to-axon diameter from MRI Both axon diameter and myelin diameter have an impact on the physical properties of nerve conduction. The ratio of internal axonal diameter to the total nerve diameter (axon+myelin) is a useful number that has theoretical relationships to axonal conduction velocity and energetic cost. This measurement is known from in vitro and histological studies of the electrical properties of axons [6]. This ratio, named the g-ratio, can be approximated as an emergent bulk tissue property using MRI.

We start by imagining a set $n$ of parallel axons. These axons are long cylinders with an internal axon radius of $r_{i n}$ and an external myelin+axonal radius of $r_{\text {out }}$. Using the cylindrical geometry, the intra-axonal space is given by $v_{i n}=n 2 \pi r_{i n}^{2} s_{\|}$ and the myelin volume by $v_{m w f}=n 2 \pi\left(r_{\text {out }}^{2}-r_{i n}^{2}\right) s_{\|}$where $s_{\|}$is a fixed axonal length. Simply taking the ratio of $v_{m w f} / v_{i n}$ yields an expression for the g-ratio, $\Gamma$ (4) in terms of the myelin volume $v_{m w f}$ and the intra-axonal volume $v_{i n}$.

$$
\Gamma=\left(\frac{v_{m w f}}{v_{i n}}+1\right)^{-\frac{1}{2}}
$$

Using only DWI or multi-component relaxometry is insufficient to estimate both $v_{i n}$ and $v_{m w f}$. To reconcile these two modalities we make use of a fourcompartment tissue model [5].

$$
S_{\text {total }}=v_{m w f} S_{1}+v_{i n} S_{2}+v_{e x} S_{3}+v_{i s o} S_{4}
$$

The last three compartments of (5) are measurable using a multi-compartment diffusion model [7]. The model allows for the estimation of the signal from multiple compartments, specifically the intra-axonal volume fraction associated with highly directional structure, $v_{i n}$. The remaining model compartment for $S_{1}$ describes signal associated, in white matter, primarily with myelin and can be estimated by T2 relaxometry. Finally, because the diffusion signal model contains no signal from $S_{1}$ it is necessary to multiply the estimates of $v_{i n}^{\prime}, v_{e x}^{\prime}$ and $v_{i s o}^{\prime}$ from the diffusion measurement (Eq. 3) by $\left(1-v_{m w f}\right)$ from (Eq. 1, Eq.2) and hence, $v_{i n}=v_{i n}^{\prime}\left(1-v_{m w f}\right)$.

Physical considerations The growth of axonal myelin is fundamental to increasing speed and efficiency of electrical activity in the brain. Myelin has a 
well-defined physical role in nerve biophysics, and the presence of myelin around an axon has a predictable and measurable effect on signal conduction velocity and energetic cost in terms of membrane charge storage $[6,9,10]$.

The conductive properties of neurones can be estimated by appealing to the physical properties of cable-like axons. Mathematically this is the solution of a partial differential equation that describes the membrane voltage change during nerve impulse propagation. Briefly, for un-myelinated axons conduction velocity $v \propto \sqrt{r_{i n}}$, whilst for myelinated nerve fibres $v \propto r_{i n} \Gamma \sqrt{-\log \Gamma}[9]$. Interestingly this expression predicts maximum conduction velocity for $\Gamma=r_{\text {in }} / r_{\text {out }}=0.61$, which, whilst born out for peripheral nerve fibres, is not found to be true in the central nervous system which has extra space and processing constraints [6].

Furthermore it is possible to derive an expression for the axonal electrostatic energetic cost, in terms of axonal charge storage requirements. The membrane charge storage and energy storage requirements are $Q=n 2 \pi r_{i n} s_{\|} C_{m} V$ and $E=\frac{1}{2} Q V$ respectively, dependent on the membrane capacitance $C_{m}$ and the membrane resting potential $V$. Given that the membrane capacitance, $C_{m}^{-1}=$ $C_{m 0}^{-1}+\left(r_{\text {out }}-r_{\text {in }}\right) C_{*}^{-1}$, for unmyelinated membrane capacitance $C_{m 0}$ and myelin layer capacitance per metre $C_{*}$ this suggests a linear relationship with $\Gamma$ since $E \propto \pi C_{*} V^{2} v_{i n} \Gamma s_{\|}$, albeit in the limit of substantial myelination ${ }^{1}$. Since the MRS NAA signal is related to increased mitochondria and hence energy turnover, we correlate the NAA/Cho ratio in the posterior white matter with the estimated g-ratio in the CST. A more accurate energetic analysis would require a compatible measurement of the axonal diameter distribution which is not currently possible in the clinical setting.

Joint Model Fitting The MRI data described above can be cast as a coupled optimisation since both models share common parameters, specifically $\Gamma$ and $v_{i s o}$. We can define the independent parameters of the DWI signal model and the T2 relaxometry as $\theta_{a}$ and $\theta_{b}$ respectively with the shared parameters as $\theta_{a b}$ Thus, the signal model for the multi-component DWI can be summarised as $S_{a}=$ $f\left(\theta_{a}=\left\{S_{a 0}, v_{i n}, \gamma, \theta, \phi\right\}, \theta_{a b}=\left\{\Gamma, v_{i s o}\right\}\right)$ and the (adult) multi-compartment T2 sequence as $S_{b}=f\left(\theta_{b}=\left\{S_{b 0}, v_{m w f}, v_{t i s s u e}\right\}, \theta_{a b}=\left\{\Gamma, v_{i s o}\right\}\right)$. This lends itself to joint non-linear optimisation with the following Jacobian matrix:

$$
\left[\begin{array}{ccc}
\frac{d S_{a}}{d \theta_{a}} & 0 & \frac{d S_{a}}{d \theta_{a b}} \\
0 & \frac{d S_{b}}{d \theta_{b}} & \frac{d S_{b}}{d \theta_{a b}}
\end{array}\right]
$$

After initialisation by the independent model-fitting, the shared parameters are jointly estimated as above for coupled non-linear least squares [11] given the concatenated signal vector $\left[S_{a}, S_{b}\right]^{T}$. The fit should be weighted by the relative modality SNRs and importantly this schema is highly general and can be used to describe any joint signal model from MRI.

\footnotetext{
${ }^{1}$ Some experimental numerical values for these parameters are available in [6].
} 


\section{Results}

Preterm cohort Figure 1 illustrates changes in $v_{m w f}$ detected in one infant at 29.4 and 40.7 weeks EGA overlaid on directional colour-coded FA images. Figure 2 plots the change in $v_{i n}, v_{m w f}$, g-ratio and NAA/Cho ratio in the ascending corticospinal tract between 28.4 and 41.7 weeks EGA. $v_{i n}$ increases significantly with gestational age $(r=0.94 p<0.001)$. The g-ratio is obtained on a voxelwise basis using Eq. 4 and we find that the mean value decreases with gestational age from an average value close to 1 at 30 weeks EGA (indicating no myelin) toward a value of 0.9 at 40 weeks EGA (Spearmans $\rho=-0.79, p=0.002$ ). We additionally correlate the CST g-ratio and $v_{i n}$ measurements with the posterior white matter MRS NAA/Cho ratio; increasing NAA/Cho correlates with increased intra-axonal content $(r=0.77 p=0.02)$ and non-significantly with g-ratio in the nearby CST $(r=-0.58 p=0.13)$ consistent with the theory that NAA change is dominated by axonal number not axonal myelination.

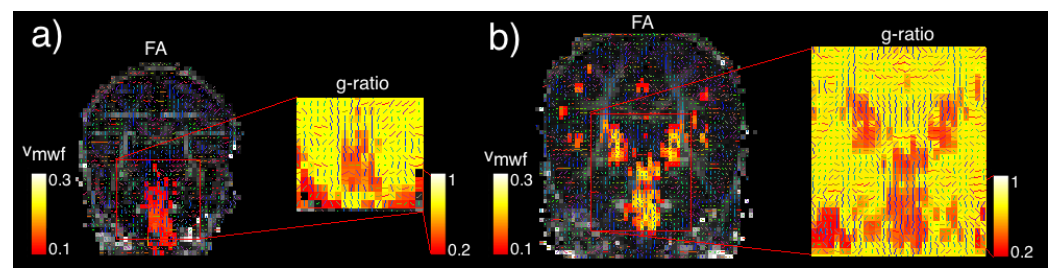

Fig. 1. Example $v_{m w f}$ maps and g-ratio maps in the same infant at 29.4 and 40.7 weeks EGA overlaid on directional FA maps. Figure best viewed electronically.
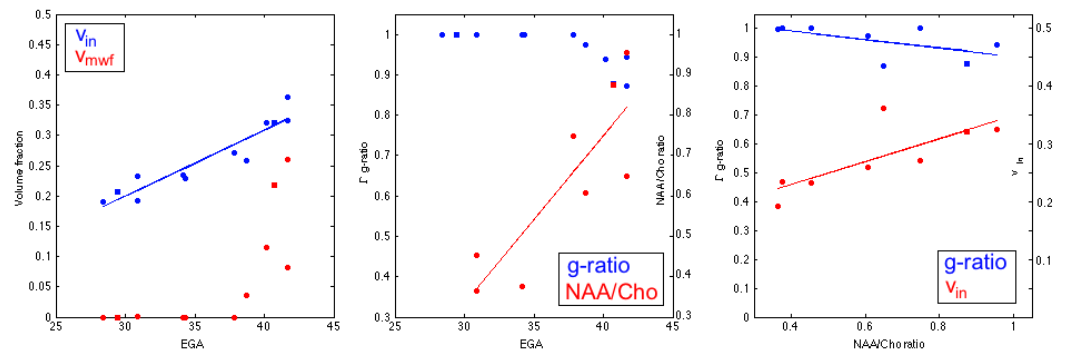

Fig. 2. Cross-sectional MR parameter changes in the corticospinal tracts of eleven preterm infants during the preterm period: a) average $v_{i n}$ and $v_{m w f}, \mathrm{~b}$ ) average gratio and NAA/Cho ratio from MRS. c) correlations of $v_{i n}^{\prime}$ and the g-ratio with the NAA/Cho ratio. Longitudinal infant data shown as squares.

Adult cohort The influence of the joint optimisation on the model fitting parameters is shown in Figure 3. The overall pattern is largely unchanged, although estimates of high $v_{i s o}$ are made more stable and estimates of the g-ratio made visually sharper. Although strictly not compatible, model fit residuals are reduced by $\approx 8 \%$ per dataset compared to the sum of independent model fit residuals.

Group average results of the seven adult datasets (after non-rigid registration to a population average space) are shown in Figure 4 . The spatial pattern of $v_{i n}$ is 
commensurate with previous results, whilst the spatial pattern of $v_{m w f}$ is largely homogenous in the white matter with the exception of regions of projective connections which have the highest $v_{m w f}$ values. The (masked) map of the g-ratio, $\Gamma$ overlaid on $v_{i n}$ shows average values of about $0.7-0.8$ throughout the white matter with values in the genu and splenium of the corpus callosum perhaps slightly less myelinated $(0.20 \pm 0.05)$ than the CST $(0.22 \pm 0.02)$. The prospective map of conduction velocity, $\Gamma \sqrt{-\log \Gamma}$, shows highest values in the CST $(0.39 \pm 0.01)$, whilst values in the corpus callosum are seen to be relatively lower $(0.34 \pm 0.04)$ despite having similar $v_{i n}(0.42 \pm 0.10$ and $0.36 \pm 0.06$ respectively $)$.

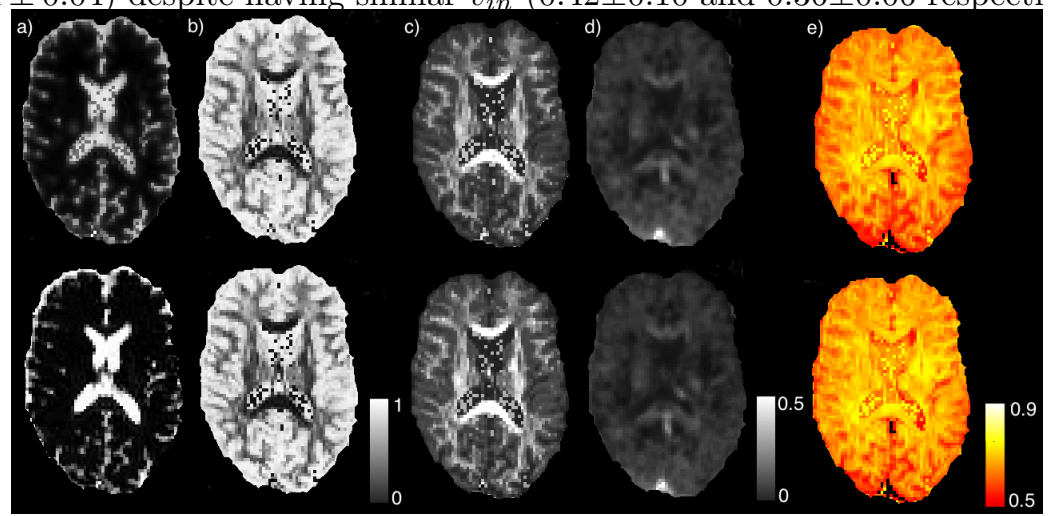

Fig. 3. Fitting adult g-ratio data using joint optimisation. Top row (columns a-e) independent model fit estimates for $v_{i s o}, \gamma, v_{i n}^{\prime}, v_{m w f}$ and $\Gamma$ Bottom row joint model fit estimates for the described parameters. Joint optimisation confers a model-fitting benefit on $v_{i s o}$ (without SNR weighting). ( $\left(v_{m w f}, \Gamma\right.$ smoothed with $2 m m$ Gaussian).

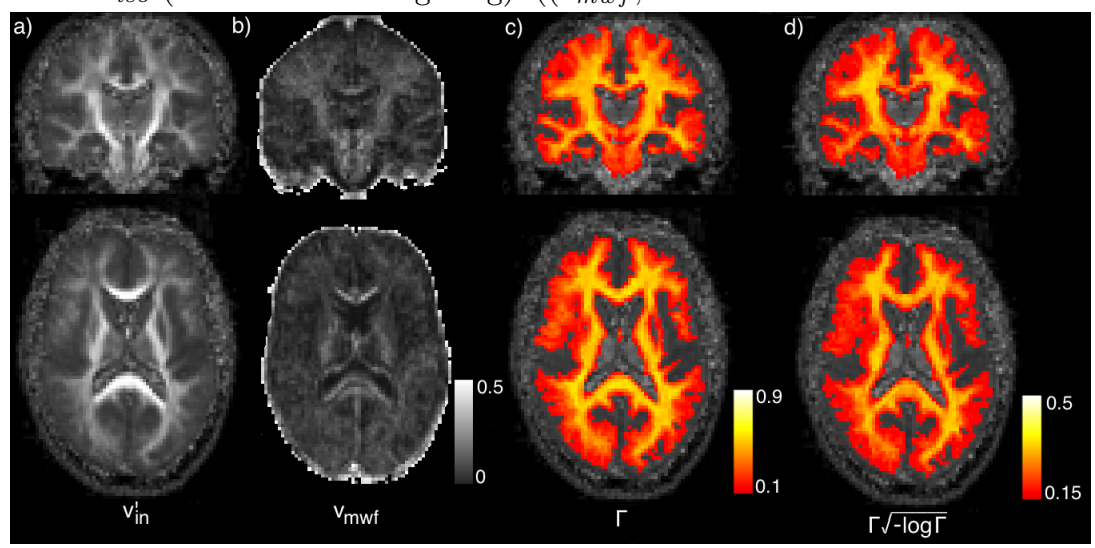

Fig. 4. Group average parameters of all 7 adult cases showing column a) $v_{i n}^{\prime}$, b) $v_{m w f}$ ) c) $\Gamma$ (expected to be proportional to the electrostatic energy cost) and d) $\Gamma \sqrt{-\log \Gamma}$ proportional to the conduction velocity (given constant axon diameter distribution). Note low $v_{i} n$ values in CST-corpus callosum intersection..

\section{Discussion}

The multi-modal data presented in this work has allowed a potential biomarker of electrical efficiency to be obtained in vivo. Although the combination of DWI 
and T2 rmakes many assumptions about the image formation process (this is also true of the individual modalities), the combination does allow new information to be obtained. Importantly, he g-ratio can be linked with simple physical models to predict the effect of change in myelin thickness on conduction velocity and energetic efficiency [6]. Future work will use this model to make predictions about functional development in preterm children. This is plausible since there is a well-defined sequence of myelination from the PLIC outward [2], and delays to this might predict corresponding delays in functional progression of motor, language and executive function as the brain increases functional electrical energetic efficiency. Irrespective of the combination of measurements in this work, the acquisition of widely available multi-shell DWI and multi-echo T2 imaging within clinically feasible time frames (preterms/adults 23mins/28mins total time) is important and will stimulate the generation of novel predictive structural biomarkers with a tangible physical link to neuronal function.

\section{References}

1. Costeloe, K.L., Hennessy, E.M., Haider, S., Stacey, F., Marlow, N., Draper, E.S.: Short term outcomes after extreme preterm birth in england: comparison of two birth cohorts in 1995 and 2006 (the epicure studies). BMJ 345 (2012) e7976

2. Brody, B.A., Kinney, H.C., Kloman, A.S., Gilles, F.H.: Sequence of central nervous system myelination in human infancy. i. an autopsy study of myelination. J Neuropathol Exp Neurol 46(3) (May 1987) 283-301

3. Stikov, N., Perry, L.M., Mezer, A., Rykhlevskaia, E., Wandell, B.A., Pauly, J.M., Dougherty, R.F.: Bound pool fractions complement diffusion measures to describe white matter micro and macrostructure. Neuroimage 54(2) (Jan 2011) 1112-1121

4. Prasloski, T., Maedler, B., B., Xiang, Q.S., MacKay, A., Jones, C.: Applications of stimulated echo correction to multicomponent t2 analysis. Magn Reson Med 67(6) (Jun 2012) 1803-1814

5. Alexander, D.C., Hubbard, P.L., Hall, M.G., Moore, E.A., Ptito, M., Parker, G.J.M., Dyrby, T.B.: Orientationally invariant indices of axon diameter and density from diffusion mri. Neuroimage 52(4) (Oct 2010) 1374-1389

6. Chomiak, T., Hu, B.: What is the optimal value of the g-ratio for myelinated fibers in the rat cns? a theoretical approach. PLoS One 4(11) (2009) e7754

7. Zhang, H., Schneider, T., Wheeler-Kingshott, C.A., Alexander, D.C.: Noddi: practical in vivo neurite orientation dispersion and density imaging of the human brain. Neuroimage 61(4) (Jul 2012) 1000-1016

8. Moffett, J.R., Ross, B., Arun, P., Madhavarao, C.N., Namboodiri, A.M.A.: Nacetylaspartate in the cns: from neurodiagnostics to neurobiology. Prog Neurobiol 81(2) (Feb 2007) 89-131

9. Matsumoto, G., Tasaki, I.: A study of conduction velocity in nonmyelinated nerve fibres. Biophysical Journal 20 (1977) 13

10. Rushton, W.A.H.: A theory of the effects of fibre size in medullated nerve. J Physiol 115(1) (Sep 1951) 101-122

11. Soo, Y.W., Bates, D.M.: Loosely coupled nonlinear least squares. Computational Statistics \& Data Analysis 14 (1992) 249-259 